\title{
Mitteilungen der DOG
}

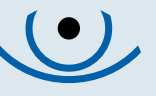

DOG

Deutsche Ophthalmologische

Gesellschaft e.V.

Die wissenschaftliche Gesellschaft

der Augenärzte

Ophthalomologe 2009 · 106:482-482

DOI 10.1007/s00347-009-1973-6

๑) Springer Medizin Verlag 2009

\section{Inhalt}

Stellungnahme der DOG zum visuellen Restitutionstraining

(VRT, Visual Restitution Training)

\section{Stellungnahme der DOG zum visuellen Restitutionstraining (VRT, Visual Restitution Training)}

\section{Februar 2009}

Das „Visual Restitution Training“ (VRT) ist ein von der Magdeburger Firma NovaVision AG für das Training von Patienten mit Gesichtsfelddefekten entwickeltes computerbasiertes Programm. Der Nutzen dieses Programms für den Patienten ist umstritten.

An der Tübinger Univ.-Augenklinik haben sich mehrere Arbeitsgruppen mit der Thematik VRT befasst und in diesem $\mathrm{Zu}$ sammenhang ein gemeinsames Untersuchungsprojekt mit der Arbeitsgruppe von Herrn Prof. B. Sabel durchgeführt: hierbei wurde bewusst das Training in den Händen dieser Arbeitsgruppe belassen - die Rekrutierung der Patienten sowie die Durchführung und Auswertung der (neuro-)ophthalmologischen Untersuchungen einschließlich der Perimetrie und SLO-Mikroperimetrie erfolgte durch Mitglieder der Tübinger Arbeitsgruppen.

Die Ergebnisse dieser Untersuchungen sind mittlerweile - unter anderem in hochrangigen Journalen mit zusätzlichen Editorials Bewertung des VRT aus (neuro)ophthalmologischer Sicht: zusammenfassend konnten die hier durchgeführten Studien mit konventionellen perimetrischen Techniken einen allenfalls minimalen Rückgang der homonymen Gesichtsfelddefekte nachweisen, der von den Autoren als irrelevant eingestuft wurde.

Auch die eigenständig von der Magdeburger Gruppe durchgeführte Auswertung wies lediglich eine durchschnittliche Verschiebung der Skotomgrenzen um „1.62 Grad" für dieses Kollektiv nach, welche das initial - gemeinsam mit der Magdeburger Arbeitsgruppe festgelegte minimale Erfolgskriterium von $2 \mathrm{Grad}$ unterschritt. Die zusätzlich durchgeführte Untersuchung mittels SLO-gestützter Mikroperimetrie unter ständiger, exakter Fixationskontrolle zeigte bei keinem der Patienten eine gleichsinnige Reduktion der homo- dieser Arbeiten ist eine skeptisc nymen Defekte des rechten und linken Auges nach Training.

Es erscheint wichtig, bei der Beurteilung derartiger Studien nicht ausschließlich nach ,statistischen Signifikanzen zu suchen“ und minimale - ggf. übungsbedingte - Effekte als Erfolg darzustellen, sondern eine alltagsrelevante Verbesserung des Sehvermögens nach VRT zu belegen. Eine solche Studie liegt nach unserem Kenntnisstand bis zum heutigen Tage nicht vor.

Das Eruieren der „subjektiven Befindlichkeit" durch Fragebögen stellt in diesem Zusammenhang nach Auffassung der Kommission keinen sinnvollen Ersatz dar, da hier ein patientenseitiger „Bias“ vorliegt: die überwiegende Mehrzahl der Patienten wird einem derart (zeit-)aufwändigen Verfahren von vorneherein einen Effekt zubilligen. Zudem zeigte sich in der gemeinsamen Studie mit den Magdeburgern kein Zusammenhang zwischen objektiven und subjektiven Daten.

Zusammenfassend ist somit aus (neuro-) ophthalmologischer Sicht anhand der in Tübingen erhobenen Daten kein Anhalt für eine alltagsrelevante Besserung des
Sehvermögens von Patienten mit homonymen Gesichtsfeldausfällen durch das Magdeburger Visual Restitution Training /VRT) gegeben.

\section{Die Deutsche Ophthalmolo- gische Gesellschaft}

Februar 2009

References "Restitution Training“

1. Schreiber A, Vonthein R, Reinhard J, Trauzettel-Klosinski S, Connert C, Schiefer U (2006) Effect of visual restitution training on absolute homonymous scotomas. Neurology 67: 143-145

2. Horton JC (2005) Vision restoration therapy: confounded by eye movements. $\mathrm{Br} J$ Ophthalmol 89: 792-794

3. Horton JC (2005) Disappointing results from Nova Vision's visual restoration therapy. Br J Ophthalmol 89. 1-2

4. Plant GT (2005) A work out for hemianopia. Br J Ophthalmol 89: 2

Reinhard J, Schreiber A, Schiefer U, Kasten E, Sabel BA, Kenkel S, Vonthein R, Trauzet tel-Klosinski S (2005) Does visual restitution training change absolute homonymous visual field defects? A fundus controlled study. Br J. Ophthalmol 89: 30-35

6. Reinhard J, Schreiber A, Schiefer U,

Vonthein R, Trauzettel-Klosinski S (2004) Visuelles Restitutionstraining bei homonymer Hemianopsie. Z Prakt Augenheilkd 25:305-312

7. Trobe, J., Sabel, B.A.,Trauzettel-Klosinski S (2005) Improving vision in a patient with homonymous hemianopia. Journal of Neuroophthalmology 25, 143-149

\section{Stellungnahme der DOG zur Orbitotomie im Rahmen der ophthalmoplastischen und rekonstruktiven Chirurgie}

Die Orbita stellt definitionsgemäß ein von knöchernen und bindegewebsartigen Strukturen begrenzten anatomischen Raum dar, dessen anteriore Begrenzung durch das Septum orbitale erfolgt.

Eine Orbitotomie liegt vor, wenn dieses als Orbita definierte Kompartiment eröffnet wird und intraorbital gelegene Weichteilstrukturen, wie das orbitale Fettgewebe, exponiert werden.
Eine Eröffnung der Orbita (Orbitotomie) erfolgt immer dann, wenn das Septum orbitale eröffnet wird; dies geschieht zum Beispiel auch im Rahmen ausgedehnter Blepharoplastiken, der Ptosis-Chirurgie sowie der Refixationen sowohl der medialen als auch lateralen Lidligamente.

März 2009 